\title{
Wir brauchen ein Primärarztsystem!
}

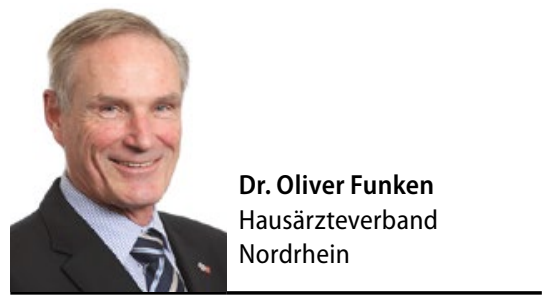

\section{Deutschlands größtes Bundesland wird bald von einer schwarz-gelben Koalition regiert. Im Wahlkampf waren einige interessante Ideen zur ambulanten ärztlichen Versorgung zu hören. Dem Hausärzteverband Nordrhein ist das nicht genug: Er fordert den großen Wurf.}

\begin{abstract}
Machtwechsel in Nordrhein-Westfalen - was können wir von der neuen Regierung aus CDU und FDP erwarten? Der designierte Ministerpräsident Armin Laschet will bis zur Sommerpause das neue Kabinett gebildet haben. In vielen Fachthemen, auch in der Gesundheitspolitik, liegen die beiden Parteien sehr nah beieinander. Was kommt nun auf uns Allgemeinmediziner zu?
\end{abstract}

Der Hausarzt spielt in der ambulanten Gesundheitsversorgung und Vorsorge eine zentrale Rolle. Alle Parteien haben ihn in ihren Programmen in den Mittelpunkt der Patientenversorgung gestellt. Wir sind die ersten Ansprechpartner für die Patienten vor Ort. Das gilt sowohl im Krankheitsfall als auch bei Fragen und Anliegen in der Prävention. Doch das gegenwärtige System trägt dem nicht genügend Rechnung.

Was wir brauchen ist ein echtes Primärarztsystem, bei dem sich der $\mathrm{Pa}$ tient bei einem Hausarzt registriert und sich mit allen gesundheitlichen Problemen zunächst an ihn wendet. Ein solches System setzt auf die gemeinschaftliche Entscheidungsfindung von Arzt und Patient. Es bietet durch die ärztliche Koordinierungsfunktion ein Mehr an Versorgungs- und Therapiesicherheit und das nachweislich ohne Verlust der Versorgungsqualität. Wir finden, dass die Teilnahme an einem solchen System den Patienten zumindest durch ein Anreizsystem erleichtert werden sollte, etwa durch geringere Beiträge zur Krankenversicherung.

\section{Auf dem Land braucht's junge Ärzte}

Ein solches attraktives System könnte mittelfristig auch dazu beitragen, dem Ärztemangel in der Stadt und im ländlichen Raum entgegenzuwirken. Auch für dieses Problem haben alle Parteien Maßnahmen vorgeschlagen. Die CDU Nordrhein-Westfalen trat dafür ein, Anreizmodelle für ärztliche Niederlassungen in unterversorgten Gebieten zu entwickeln. In ländlichen Regionen soll die Arbeit für Nachwuchsärzte so attraktiv und wirtschaftlich gestaltet sein, dass sie nach dem Studium eine Praxis übernehmen wollen.
Mehr Hausärzte erhofft sich die CDU auch von einer neu zu schaffenden medizinischen Fakultät in Ostwestfalen-Lippe. Alle Landtagsparteien forderten im Wahlkampf die Stärkung der medizinischen Fakultäten und speziell des Fachs Allgemeinmedizin. Mehr Studienplätze und eine bessere Vergabe sollen zudem mehr junge Menschen für das Medizinstudium gewinnen.

Das alles ist jedoch aus unserer Sicht noch zu wenig.

Es ist unabdingbar, an allen Universitäten in NRW leistungsstarke, den anderen Lehrstühlen gleichberechtigte Lehrstühle für Allgemeinmedizin mit engagierten Professoren zu installieren. Das Studium muss frühzeitig praxisnah und breit aufgestellt sein. Außerdem brauchen wir verpflichtende Zeiten der Weiterbildung in der Allgemeinmedizin, um den Studierenden die komplexe Denkweise des Fachs am Patienten nahezubringen. Nur so wird es gelingen, Studierende für den Beruf Hausarzt zu begeistern.

Im Sommer wird die neue Regierung politisch Fahrt aufnehmen. Gespannt sind wir, wer Verantwortung in der Gesundheitspolitik übernimmt. Veränderung bringt immer Bewegung mit sich. Wir Hausärzte sind startbereit!

- Der Autor ist Hausarzt aus Rheinbach und 2. Vorsitzender des Hausärzteverbands Nordrhein 